\title{
Diacronie
}

Studi di Storia Contemporanea

$\mathrm{N}^{\circ} 29,1$ | 2017

"Crash test"

\section{Un oscuro protagonista dell'affaire Moro: Antonio Chichiarelli e il falso comunicato n. 7}

\section{Francesco Landolfi}

\section{(2) OpenEdition}

\section{Journals}

\section{Edizione digitale}

URL: http://journals.openedition.org/diacronie/5162

DOI: 10.4000/diacronie.5162

ISSN: 2038-0925

\section{Editore}

Association culturelle Diacronie

\section{Notizia bibliografica digitale}

Francesco Landolfi, « Un oscuro protagonista dell'affaire Moro: Antonio Chichiarelli e il falso comunicato n. 7 », Diacronie [Online], № 29, 1 | 2017, documento 3, Messo online il 29 mars 2017, consultato il 20 avril 2019. URL : http://journals.openedition.org/diacronie/5162 ; DOI : 10.4000/ diacronie. 5162 


\section{Diacronie}

Studi di Storia Contemporanea

29, 1/2017

"Crash test". Continuità, discontinuità, legami e rotture nelle dinamiche della storia contemporanea

\section{Un oscuro protagonista dell'affaire Moro: Antonio Chichiarelli e il falso comunicato n. 7}

Francesco LANDOLFI

Per citare questo articolo:

LANDOLFI, Francesco, «Un oscuro protagonista dell'affaire Moro: Antonio Chichiarelli e il falso comunicato n. 7», Diacronie. Studi di Storia Contemporanea : "Crash test". Continuità, discontinuità, legami e rotture nelle dinamiche della storia contemporanea, 29, 1/2017, 29/3/2017,

URL: < http://www.studistorici.com/2017/03/29//andolfi_numero_29/ >

Diacronie Studi di Storia Contemporanea $\rightarrow$ http://www.diacronie.it

Rivista storica online. Uscita trimestrale.

redazione.diacronie@hotmail.it

Comitato di direzione: Naor Ben-Yehoyada - João Fábio Bertonha - Christopher Denis-Delacour - Maximiliano Fuentes Codera Anders Granås Kjøstvedt - John Paul Newman - Deborah Paci - Niccolò Pianciola - Spyridon Ploumidis - Wilko Graf Von Hardenberg

Comitato di redazione: Jacopo Bassi - Luca Bufarale - Gianluca Canè - Fausto Pietrancosta - Alessandro Salvador - Matteo Tomasoni Diritti: gli articoli di Diacronie. Studi di Storia Contemporanea sono pubblicati sotto licenza Creative Commons 3.0. Possono essere riprodotti e modificati a patto di indicare eventuali modifiche dei contenuti, di riconoscere la paternità dell'opera e di condividerla allo stesso modo. La citazione di estratti è comunque sempre autorizzata, nei limiti previsti dalla legge. 


\title{
3/ Un oscuro protagonista dell'affaire Moro: Antonio Chichiarelli e il falso comunicato n. 7
}

\author{
Francesco LANDOLFI
}

Il sequestro dell'onorevole democristiano Aldo Moro da parte delle Brigate rosse ha rappresentato il momento più drammatico della storia della Repubblica Italiana. La lotta contro le istituzioni trascinò così la nazione in una situazione di imminente guerra civile, determinando nell'animo della popolazione un quotidiano senso di terrore. In questo specifico episodio emerge la figura criminale di Antonio Chichiarelli: simpatizzante brigatista, neofascista, informatore, falsario della banda della Magliana ed esecutore materiale del falso comunicato n. 7. Lo scopo del saggio è evidenziare i possibili legami operativi che potrebbero essere avvenuti tra la malavita romana, le Brigate rosse e le agenzie di sicurezza all'interno di una vicenda ancora oggi pervasa da numerose questioni irrisolte.

\section{La vicenda}

Giovedì 16 marzo 1978 il presidente della Democrazia cristiana (DC), Aldo Moro, lasciò in automobile la sua abitazione in via Forte Trionfale 79 per dirigersi, insieme ai cinque agenti della scorta, in piazza Montecitorio. L'appuntamento cui non poteva mancare era il frutto del suo lavoro politico degli ultimi anni: l'apertura della maggioranza parlamentare democristiana al Partito comunista italiano (PCI) di Enrico Berlinguer. Sembrò che il progetto del "compromesso storico" stesse per realizzarsi. Quella mattina, infatti, la Camera dei Deputati avrebbe votato la fiducia per il quarto governo presieduto da Giulio Andreotti dopo un accordo programmatico elaborato anche dal PCI. Mentre percorreva via Mario Fani, alle $9.02^{1}$ la vettura FIAT 130 dell'on. Moro venne bloccata da una FIAT 128 bianca con targa del Corpo diplomatico, che gli si posizionò di fronte. Dopo il massacro

\footnotetext{
${ }^{1}$ La mattina del 16 marzo 1978, nei pressi di via Fani è stata accertata la presenza del colonnello del Servizio d'Informazione per la Sicurezza Militare (SISMI) Camillo Guglielmi, già addestratore per le tecniche d'imboscata nella base militare di Gladio a Capo Marrangiu (SS), oltre che di Bruno Barbaro, cognato del colonnello Fernando Pastore Stocchi, anche lui dirigente del centro di addestramento sardo. Cfr. X LEGISLATURA, Commissione parlamentare d'inchiesta sul terrorismo in Italia e sulle cause della mancata individuazione dei responsabili delle stragi, Relazione sull'inchiesta condotta sugli ultimi sviluppi del caso Moro, 22 aprile 1992, Doc. XXIII, n. 49, p. 40.
} 
dei cinque uomini della scorta, il commando terrorista delle Brigate rosse (BR) si dileguò sequestrando il politico ${ }^{2}$.

Secondo un'interessante ricerca condotta dai giornalisti Romano Bianco e Manlio Castronuovo, furono almeno ventiquattro i testimoni oculari che assistettero alla sparatoria. Uno di questi, Pietro Lalli, riconobbe in uno degli assassini una persona particolarmente addestrata nell'utilizzo delle armi, perché notò la maniera disinvolta con cui «impugnava il mitra [...], tenendo la mano sinistra sulla canna per evitare che si impennasse» 3 e apparendo allo stesso tempo freddo e deciso nei movimenti. Atteggiamenti propri di un militare e non di un terrorista. A questo killer, peraltro, gli studi topografici e balistici attribuirono 49 proiettili degli 89 totali sparati dai brigatisti. Questione ancora più incredibile fu il fatto che 31 bossoli ritrovati sul luogo del delitto avrebbero potuto essere utilizzati soltanto da organi militari «non convenzionali»4. Una tipologia di munizioni presente solamente all'interno dei depositi militari segreti dell'organizzazione paramilitare Gladio.

Nonostante la rilevanza delle forze impiegate (172.270 agenti e 21.399 automezzi), delle attività svolte (6.296 posti di blocco, 17.756 pattugliamenti e 6.933 perquisizioni domiciliari) e dei controlli attuati (167.109 su persone e 96.572 su automezzi) dagli organi della pubblica sicurezza all’interno dell'area metropolitana di Roma5, il 9 maggio il cadavere di Moro, colpito da undici proiettili (di cui dieci esplosi con un mitra Browning 7.65 modello Skorpion), venne ritrovato nel bagagliaio di un'automobile Renault 4 rossa abbandonata in via Caetani. Una via centrale di Roma, a metà strada tra la sede della DC e quella del PCI 6 .

L'eliminazione di Moro dalla scena politica si verificò proprio nello stesso periodo in cui lo statista stava perseguendo una concreta alleanza di governo con il Partito comunista. Una mossa strategica fortemente osteggiata dal governo statunitense7. Già dalla fine del 1968 Moro espresse apertamente al proprio partito la volontà di collaborare con l'opposizione comunista, al fine di garantire un governo stabile tra il PCI e la DC. Tale convergenza politica, definita come «strategia dell'attenzione»8, risultò

\footnotetext{
${ }^{2}$ VIII LEGISLATURA, Commissione parlamentare d'inchiesta sulla strage di via Fani sul sequestro e l'assassinio di Aldo Moro e sul terrorismo in Italia. Relazione conclusiva, 29 giugno 1983, Vol. I, Doc. XXIII, No. 5, p. 10.

${ }^{3}$ BIANCO, romano, CASTRONUOVO, Manlio, Via Fani ore 9.02. 34 testimoni oculari raccontano l'agguato ad Aldo Moro, Roma, Nutrimenti, 2010, p. 59.

${ }^{4}$ BIONDO, Nicola, Una primavera rosso sangue. I documenti ufficiali sull"'Affaire Moro". Gli attori, i protagonisti, le fazioni ancora in lotta, Cosenza, Memoria, 1998, p. 38.

${ }^{5}$ VIII LEGISLATURA, Commissione parlamentare d'inchiesta, cit., p. 36.

${ }^{6}$ SUNDQUIST, Victor H., «Political Terrorism: An Historical Case Study of the Italian Red Brigades», in Journal of Strategic Security, 3, 3/2010, pp. 53-68, p. 60.

${ }^{7} \mathrm{Nel}$ settembre del 1974 un collaboratore del segretario di stato americano Henry Kissinger minacciò severamente Moro della pericolosità di tale legame col PCI: «Onorevole, lei deve smettere di perseguire il suo piano politico di portare tutte le forze del suo Paese a collaborare direttamente. O lei smette di fare questa cosa, o la pagherà cara». Il 13 settembre 1975 il settimanale «OP-Osservatore Politico» del giornalista Carmine Pecorelli, sotto il titolo L'America esperta scherza e prevede, aveva riportato una frase profetica, pronunciata da un funzionario al seguito di John Ford, in visita a Roma: «Vedo nero. C'è una Jacqueline nel futuro della vostra penisola». Presto qualcuno avrebbe fatto la stessa fine del presidente John F. Kennedy. Cfr. FLAMIGNI, Sergio, Le idi di marzo. Il delitto Moro secondo Mino Pecorelli, Milano, Kaos, 2006, p. 151. D'altra parte, l'onorevole Moro aveva parlato con Vittorio Cervone, parlamentare democristiano e suo amico intimo, presagendogli un triste futuro: "You will see, they will make us pay for our political line». Cfr. PASCALI, Umberto, «New Evidence of Kissinger's Role in Aldo Moro Murder», in Executive Intelligence Review International, 9, 31, 1982, pp. 34-35, p. 35. ${ }^{8}$ SCIRE, Giambattista, «Il caso Moro. Frammenti di una verità indicibile», in Italia contemporanea, 255, giugno 2/2009, pp. 273-305, p. 278.
} 
chiaramente agli occhi degli Stati Uniti e da Kissinger come l'inizio di una possibile spaccatura interna al blocco occidentale. Su tale questione il punto di vista del Dipartimento di Stato americano sembrò essere stato piuttosto chiaro e netto. Il comunicato del 12 gennaio 1978 del Pentagono alle istituzioni italiane non lasciò dubbi su come si sarebbe dovuto agire:

Esperti del Governo hanno ripetutamente espresso tali vedute sulla questione della partecipazione dei comunisti ai governi dell'Europa occidentale. La nostra posizione è chiara: noi non siamo favorevoli a tale partecipazione e vorremmo veder diminuire l'influenza comunista nei paesi dell'Europa occidentale. Come abbiamo detto in passato, riteniamo che il modo migliore per conseguire questi obiettivi sia attraverso gli sforzi dei partiti democratici per soddisfare le aspirazioni popolari di un governo efficiente, giusto e aperto alle istanze sociali9.

Si trattò della cosiddetta "dottrina Kissinger", secondo la quale i governi democratici (in particolare quello italiano) avrebbero dovuto seguire una linea politica di intransigente condanna nei confronti dei partiti comunisti. Nata alla fine degli anni sessanta quando Kissinger divenne Consigliere per la sicurezza nazionale durante la presidenza di Richard Nixon, questa strategia diplomatica d'intolleranza verso il blocco sovietico si originò a partire dal quadro internazionale che ormai fin dalla crisi della guerra in Vietnam sembrava instabile per gli Stati Uniti. A ciò si aggiunsero ulteriori questioni legate alle rivolte sociali (fenomeno del Black Panther Party nel 196973), all'economia (svalutazione del dollaro nell'agosto 1971, crisi energetica nell'ottobre 1973) e alle istituzioni (scandalo Watergate durante il biennio 1972-74), che indebolirono la supremazia mondiale americana. Per tutti gli anni settanta la "dottrina Kissinger" divenne il principio cardine da seguire non soltanto per la diplomazia statunitense, ma anche per l'intero blocco occidentale, soprattutto dopo il cambiamento politico verificatosi in Cile nel novembre 1970 con l'avvento del socialista Salvador Allende e nell'area mediterranea con la fine delle dittature militari filoamericane in Grecia, Spagna e Portogallo nel 1974-75.

In tale contesto di generale crisi l'Italia si pose come un paese proiettato verso una politica progressista favorevole alle nuove esigenze popolari, generatesi dal periodo di crisi economica, politica e sociale cominciato con l'"l'autunno caldo" del 1969, preludio della parentesi temporale che la storia ha definito "gli anni di piombo". L'esplosione di quello che lo storico economico Pierluigi Ciocca definisce come un triplice shock (salariale, petrolifero, finanziario pubblico) produsse nella penisola un aumento della disoccupazione e del costo della vita, insieme a una contemporanea svalutazione della lira, che durante la prima metà degli anni settanta incrementò la percentuale del debito pubblico nazionale di 20 punti, passando dal 40 al 60\% rispetto alla cifra totale del PIL ${ }^{10}$.

\footnotetext{
${ }^{9}$ MAGLiOCCO, Mario, Stati Uniti e Pci 1943-1980, Roma-Bari, Laterza, 1981, p. 280.

${ }^{10}$ CIOCCA, Pierluigi, Ricchi per sempre? Una storia economica d'Italia (1796-2005), Torino, Bollati Boringhieri, 2007, pp. 285, 291 (fig. 11.3)
} 
In questo periodo la politica democristiana applicò una serie di riforme socio-istituzionali che modificarono profondamente il paese: lo Statuto dei lavoratori (1970) e l'istituzionalizzazione delle regioni (1970) misero in pratica la "seconda via" del riformismo socialdemocratico concretizzatosi con il primo governo Moro nel dicembre 1963, inglobando all'interno del governo di maggioranza democristiano anche il Partito socialista italiano (PSI) di Giuseppe Saragat e Pietro Nenni in funzione anticomunista. Una fase di mutamento istituzionale che era stata prevista dalle agenzie di sicurezza statunitensi già nel 1961:

Moro [...] is convinced that support of a pro-western foreign policy will eventually involve the PSI in polemics with communists (PCI) and thereby cause a de facto split. [...] Moro is convinced that after five years of a center-left government the PSI will cease to exist as a political power. Some two thirds of the PSI would be won over to the DC position and the other third would move to the $\mathrm{PCI}^{11}$.

La strategia della "seconda via" intrapresa da Moro e dalla DC risultò consimile a quella formula che Kennedy definì come la New Frontier, una nuova fase storica finalizzata alla distensione con l'Unione Sovietica e al disarmo reciproco degli armamenti nucleari. Questo significò in Italia la possibilità dell'avvento di un governo socialdemocratico attraverso un avvicinamento della DC con il PSI: «President Kennedy called this fresh approach to the New Frontier. We call it the less appealing name of center-left» ${ }^{12}$. Lo spostamento a sinistra delle istituzioni democristiane e il notevole consenso che gli italiani continuarono a mostrare verso il Partito comunista italiano preoccuparono gli Stati Uniti per la possibilità d'incorrere in una "terza via" di governo con il PCI che in un futuro prossimo sarebbe stata inevitabile. Nonostante ciò, ancora nel 1965 gli Usa confidarono ancora pienamente nella volontà di Moro di estromettere eventuali ingerenze comuniste all'interno della coalizione socialdemocratica: «Majority of DC (including Moro) have no illusions concerning nature of PCI and threat it constitutes for Italian democracy» ${ }^{13}$.

Eppure con la fine del suo terzo governo nel giugno 1968, Moro cominciò ad avvicinarsi al Partito comunista. Questa rischiosa manovra politica venne giustificata dallo statista democristiano dall'analisi del cambiamento socio-culturale che si era verificato nella penisola e nel mondo in seguito alla sconfitta militare americana in Vietnam e alle conseguenti rivolte studentesche e operaie sfociate nell'eclatante "maggio parigino". Ciò aveva posto in discussione la supremazia americana, che non si configurava più come una terra di libertà e democrazia, ma come un impero

\footnotetext{
${ }^{11}$ CENTRAL INTELLIGENCE AGENCY (CIA), Moro's Views on Future Developments in the Italian Political Situation, No. IDCS-3/491,321, 27 ottobre 1961, p. 3.

${ }^{12}$ CIA, Moro's Comments on the Italian Political Situation, No. CS-3/521-157, agosto 1962, p. 5.

${ }^{13}$ FOREIGN RELATIONS OF THE UNITED STATES (FRUS) 1964-1968 WESTERN EUROPE, Telegram From the Embassy in Italy to the Department of State, Vol. XII, Doc. 108, No. 2659, Roma, 9 aprile 1965, p. 227.
} 
consumistico-capitalista. Una lungimirante riflessione che Moro espose chiaramente in un suo discorso durante il Consiglio Nazionale democristiano, delineante la sua ferma intenzione di rinnovare la DC attraverso un avvicinamento al PCI:

Tempi nuovi si annunciano ed avanzano in fretta come non mai. Il vorticoso succedersi delle rivendicazioni, la sensazione che storture, ingiustizie, zone d'ombra, condizioni d'insufficiente dignità e di insufficiente potere non siano oltre tollerabili, l'ampliarsi del quadro delle attese e delle speranze all'intera umanità, la visione del diritto degli altri, anche dei più lontani, da tutelare non meno del proprio, il fatto che i giovani, sentendosi ad un punto nodale della storia, non si riconoscano nella società in cui sono e la mettano in crisi, sono tutti segni di grandi cambiamenti e del travaglio doloroso nel quale nasce una nuova umanità.

[...] Farò ora alcune considerazioni sui problemi del partito. È mio dovere comunicarvi, come ho fatto da tempo al Segretario politico, che ho deciso di assumere una posizione autonoma nella organizzazione interna della Democrazia cristiana, autonomia che eserciterò, specie nell'attuale contingenza, con quel senso di responsabilità che ha sempre caratterizzato la mia azione ${ }^{14}$.

Nixon e Kissinger mostrarono un'immediata preoccupazione verso l'atteggiamento progressista di Moro, cominciando peraltro a pronosticare un ingresso del PCI nel governo verso la metà degli anni settanta. Come mossa preventiva venne inviato a Roma un nuovo ambasciatore statunitense, Graham Martin, diplomatico intransigente che durante i suoi quattro anni di carica (1969-1973) rifiutò costantemente qualsiasi confronto con Moro riguardo all'incerta situazione politica italiana ${ }^{15}$. Intanto Moro, divenuto Ministro degli Esteri nel secondo governo presieduto dal democristiano Mariano Rumor, continuò a rassicurare il governo statunitense in merito all'impossibilità di una coalizione con il PCI: «Moro promised he would remain in close touch with US as Italian position developed as he did not desire to take any action which would be harmful to US interests ${ }^{16}$. Dal punto di vista sociale gli anni che seguirono furono contraddistinti dallo sviluppo del terrorismo politico e del fenomeno stragista, inauguratosi con la bomba alla Banca dell'Agricoltura in piazza Fontana a Milano, che il 12 dicembre 1969 provocò la morte di diciassette persone e il ferimento di altre ottantotto. Uno strisciante clima di guerra civile e di destabilizzazione socio-politica che, secondo quanto scritto dall'ex agente CIA Philip Agee, può talvolta essere provocato ad hoc, in base alla metodologia dei servizi segreti, per favorire un ripristino delle istituzioni democratiche attraverso una già menzionata «strategia della tensione»:

\footnotetext{
${ }^{14}$ MORO, Aldo, Intervento al Consiglio Nazionale della Dc, Roma, 21 novembre 1968.

${ }^{15}$ FORMIGONI, Guido, Aldo Moro. Lo statista e il suo dramma, Bologna, Il Mulino, 2016, p. 236.

${ }^{16}$ FRUS 1969-1976 WESTERN EUROPE, NATO 1969-1972, Telegram From the Embassy in Italy to the Department of State, Vol. XLI, Doc. 182, No. 5557, Roma, 2 settembre 1969, p. 632.
} 
Se una situazione può essere più efficacemente recuperata agli interessi americani con metodi non costituzionali o attraverso un colpo di Stato, può essere tentata anche questa via. Anche se in genere l'Agenzia [CIA] per montare un colpo di Stato gioca la carta dell'anticomunismo, possono servire ugualmente bene allo scopo anche i lingotti d'oro e i sacchi di banconote. In alcuni casi una bomba opportunamente fatta esplodere da un agente della sezione, seguita da dimostrazioni di massa e poi dall'intervento dei militari in nome del ristabilimento dello ordine e dell'unità nazionale, può essere la strada più conveniente ${ }^{17}$.

In proposito alle elezioni politiche del 20 giugno 1976, la CIA espresse tutta la propria preoccupazione riguardo all'instabile situazione politica italiana che sembrava favorire il PCI, a scapito di una maggioranza socialdemocratica ormai in declino. In questo spirito d'insicurezza istituzionale gli statunitensi ritennero «essenziale» ${ }^{18}$ un rafforzamento deciso e immediato del potere democristiano, in previsione di un momento di sorpasso dei comunisti sulla DC:

Last June [1975] the Communists captured about a third of the vote in nationwide regional and local elections, coming within 2 percent of the Cristian Democrats. This gave the Communist question a new immediacy, deepened existing divisions within the governing parties, and dealt the final blow to the foundering center-left alliance that had governed Italy since 1963.

[...] The last year was the most successful in the Communist Party's history. As a result of the party's gains in the local elections, 60 to 65 percent of the Italian population is now governed by regional or local administrations dominated by or dependent on the Communists.

In addiction, The Communists are operating in a far more receptive political atmosphere than at any previous time ${ }^{19}$.

Nel 1976 il PCI ottenne il 34\% dei voti e le speranze del «compromesso storico» voluto da Berlinguer e da Moro divennero sempre più concrete. Le relazioni internazionali tra Roma e Mosca continuarono a migliorare, sebbene il Cremlino divergesse politicamente da Berlinguer in merito al fatto che PCI avesse autonomamente deliberato di entrare in un governo di coalizione democristiano. Un'alleanza politica che sostanzialmente non fu apprezzata in toto né dagli Stati Uniti né dall'Unione Sovietica. È anche in tale contesto storico che deve essere inserito il sequestro e l'assassinio di Aldo Moro ${ }^{20}$.

Nel corso dei cinquantacinque giorni intercorsi tra il 16 marzo e il 9 maggio 1978 i servizi segreti italiani, diretti dai generali Giuseppe Santovito, Giulio Grassini e dal prefetto di Roma Walter Pelosi, furono allo stesso tempo responsabili di una serie di inefficienze e di errori difficilmente ascrivibili

\footnotetext{
${ }^{17}$ AGEE, Philip, Agente della CIA, Roma, Editori Riuniti, 1975, p. 91.

${ }^{18}$ CIA, The President's Daily Brief, 10 giugno 1976, p. 6.

${ }^{19}$ CIA, The President's Daily Brief, 17 maggio 1976, pp. A1-A3.

${ }^{20}$ ANDREW, Christopher, GORDIEVSKIJ, Oleg, La storia segreta del KGB, Milano, Rizzoli, 2005, pp. 639-640.
} 
alla pura e semplice casualità. Sintomatici, al contrario, di una precisa operazione di depistaggio e di occulta protezione dell'azione terroristica. Non sembra casuale il fatto che il Comitato di crisi, istituito al Viminale dal ministro dell'Interno Francesco Cossiga, fosse composto interamente da infiltrati della Loggia P2, ingaggiati quali esperti o consiglieri del ministro ${ }^{21}$.

I giorni del sequestro vennero scanditi da periodici comunicati firmati Brigate rosse. Numerati progressivamente, furono dieci in tutto, prima del fatidico 9 maggio ${ }^{22}$. I comunicati vennero sempre divulgati dai brigatisti seguendo un'identica prassi, caratterizzandosi «per gli identici aspetti tecnici, filologici e ideologici, anche allo scopo di eludere eventuali “ingerenze esterne" nella gestione del sequestro» ${ }^{23}$. Tuttavia, di questi dieci se ne riscontrò uno che presentava caratteristiche completamente diverse dai precedenti: molto sintetico, scritto con uno stile satirico su un foglio più corto del solito, senza slogan conclusivi e, infine, con l'intestazione «BRIGATE ROSSE» scritta stranamente a mano ${ }^{24}$. Si trattò del falso comunicato n. 7 del 18 aprile 1978. Documento scritto da un criminale della banda della Magliana: "Tony" Chichiarelli.

\section{Il personaggio}

Antonio Giuseppe Chichiarelli nacque in un paesino dell'appennino abruzzese, Rosciolo, frazione di Magliano dei Marsi, il 16 gennaio 1948. Dopo aver espletato il servizio di leva nel corpo degli Alpini ed essere rimasto affascinato dalle prime agitazioni studentesche presso le Università di Pisa, Trento e Torino, decise di trasferirsi nella capitale ${ }^{25}$. Fin dai primi anni vissuti a Roma Chichiarelli ebbe problemi con la giustizia; nel 1970 venne fermato per detenzione di armi. Tre anni dopo venne rinchiuso per un breve periodo nel carcere di Regina Coeli per ricettazione ${ }^{26}$. In quegli anni conobbe Luciano Dal Bello, criminale romano legato alla banda della Magliana e dal 1977 anche informatore del Servizio d'Informazione per la Sicurezza Democratica (SISDE) e dei carabinieri del Nucleo polizia giudiziaria e del Nucleo tutela patrimonio artistico di Roma. Nel 1976 subì un secondo arresto e in

\footnotetext{
${ }^{21}$ X LEGISLATURA, Commissione parlamentare d'inchiesta, cit., pp. 32-33. Tra questi si ricordano Federico Umberto D’Amato (fiduciario dei servizi segreti atlantici), lo psichiatra Franco Ferracuti (in rapporti con la CIA), il prefetto Fernando Guccione (responsabile della Sala Situazione Globale istituita da Cossiga) e l'ammiraglio Antonio Geraci (capo del SiOS della marina). Cfr. Sergio Flamigni, La loggia P2, in Luciano Violante (a cura di), Storia d'Italia, La criminalità, Annali, Vol. 12, Torino, Einaudi, 1997, p. 444.

${ }^{22}$ I dieci comunicati brigatisti, compreso quello falso del Lago della Duchessa, possono essere consultati in BOCCA, Giorgio (a cura di), Moro: una tragedia italiana, Milano, Bompiani, 1978.

${ }^{23}$ FLAMIGNI, Gianni, La banda della Magliana. Storia di una holding politico-criminale, Milano, Kaos, 2002, p. 35.

${ }^{24}$ FLAMIGNI, Gianni, La sfinge delle Brigate rosse. Delitti, segreti e bugie del capo terrorista Mario Moretti, Milano, Kaos, 2004, pp. 272-273.

${ }^{25}$ BIONDO, Nicola, VENEZIANI, Massimo, Il falsario di Stato. Uno spaccato noir della Roma degli anni di piombo, Roma, Cooper, 2008, pp. 23-24.

${ }^{26}$ Secondo Dal Bello, Chichiarelli si riforniva di armi presso una base aerea Nato vicino Napoli. È possibile che molte di quelle armi possano essere state utilizzate dalla banda della Magliana, visto che Chichiarelli era solito «affitta[re] armi alla malavita romana». Cfr. FLAMIGNI, Gianni, La tela del ragno. Il delitto Moro, Roma, Edizioni Associate, 1988, pp. 196, 198.
} 
quell'occasione conobbe Danilo Abbruciati, che pochi anni dopo sarebbe emerso come uno dei leader della banda della Magliana ${ }^{27}$.

A parte il suo coinvolgimento nel mondo criminale, Chichiarelli dimostrò di possedere un innato talento per la pittura. Si specializzò nella riproduzione di quadri famosi. Un'attività, quella dei "falsi autentici", situata in una linea di confine tra il mondo della legalità e quello dell'illegalità. Parallelamente al falso d'autore, Chichiarelli non disdegnò di occuparsi ad un secondo "lavoro": la falsificazione di documenti.

Chichiarelli dimostrò di possedere uno spirito eclettico anche nell'attivismo politico. Manifestò simpatie per l'estrema sinistra extraparlamentare, frequentando tra il 1976 e il 1977 gli ambienti legati ad Autonomia operaia. Eppure, allo stesso tempo non disdegnò di incontrarsi con personaggi che si dichiararono fascisti convinti, come i boss della banda della Magliana Franco Giuseppucci e Abbruciati, o il neofascista dei Nuclei armati rivoluzionari Massimo Sparti ${ }^{28}$.

Questa fu la vita di Antonio Chichiarelli fino a circa due mesi prima del sequestro Moro. Tra la fine del 1977 e l'inizio del 1978 il falsario venne coinvolto nella stesura del falso comunicato brigatista che avrebbe dovuto annunciare l'avvenuta morte di Moro e il suo occultamento nel lago della Duchessa.

In realtà, autorevoli periti grafici nelle loro relazioni giunsero a un'inaspettata conclusione: furono ben tre i comunicati cui vennero attribuite caratteristiche simili tra loro e che contemporaneamente si differenziarono dagli altri. Si trattò quindi del primo comunicato emesso subito dopo il sequestro (al quale proprio le Brigate rosse raccomandavano di rapportarsi per verificare l'autenticità dei successivi comunicati), del ben noto "falso" settimo comunicato (definito dai brigatisti come «una lugubre mossa degli specialisti della guerra psicologica» ${ }^{29}$ e attribuito a Chichiarelli) e del decimo comunicato fatto ritrovare soltanto il 20 maggio (anche questo non rivendicato dalle Brigate rosse). Se il primo comunicato, considerato come il termine di paragone per verificare l'autenticità degli altri messaggi seguenti, fu redatto con la stessa macchina utilizzata per scrivere il settimo, allora è plausibile che Chichiarelli abbia potuto redigere anche il primo comunicato, quello "vero" per antonomasia. Di conseguenza, si potrebbe pensare che il falsario avrebbe potuto quantomeno risultare in contatto con le Brigate rosse durante i giorni del sequestro Moro. A tal proposito scrisse nel novembre 1990 l'avvocato Giuseppe Zupo (dirigente del settore

\footnotetext{
${ }^{27}$ X LEGISLATURA, Commissione parlamentare d'inchiesta, cit., p. 39.

${ }^{28}$ CORTE D’ASSISE DI PERUGIA (CAP), Sentenza di primo grado, 24 settembre 1999, pp. 253-254. Sparti è colui che fornì a Valerio Fioravanti e Francesca Mambro i documenti falsi dopo la strage del 2 agosto 1980 alla stazione di Bologna.

${ }^{29}$ Il vero settimo comunicato brigatista del 20 aprile 1978 è consultabile in BOCCA, Giorgio (a cura di), Moro, cit., p. 126. Il volantino venne annunciato poco dopo le 12.00 del 20 aprile 1978 con una telefonata a «Il Messaggero». Il documento venne fatto ritrovare a Roma in via dei Maroniti, dietro la sede della testata giornalistica romana, dentro una busta arancione contenente anche una foto Polaroid di Moro con in mano una copia de «La Repubblica» del 19 aprile, prova evidente che era ancora vivo. Il comunicato venne in seguito diffuso anche a Torino, Genova e Milano.
} 
giustizia del PCI e legale delle famiglie degli uomini della scorta trucidata di Moro) in un appunto per la segreteria del suo partito:

È chiaro dunque che se la testina rotante o la macchina servita per scrivere i comunicati $\mathrm{Br}$ durante il sequestro, a partire dal più significativo e reclamizzato di essi, il n. 1, erano in possesso di gente come quella coinvolta con la banda della Magliana, l'operazione Moro è riconducibile provatamente a livelli diversi da quelli noti del terrorismo rosso. Livelli in cui servizi e alta criminalità anche finanziaria sono documentalmente protagonisti30.

Una testina rotante per macchina da scrivere IBM venne addirittura trovata in possesso di Chichiarelli nell'agosto 1979 da parte degli agenti di polizia del commissariato Monteverde. Incredibilmente, dopo generiche indagini, questa fu subito restituita al proprietario ${ }^{31}$.

\section{Il documento}

Alle ore 9,25 del 18 aprile 1978, il trentaquattresimo giorno del sequestro, una telefonata anonima giunse alla redazione del quotidiano romano «Il Messaggero», annunciando che all'interno di un cestino in piazza Gioacchino Belli a Roma si sarebbero trovati due messaggi delle BR. In realtà venne ritrovato un solo volantino, contenuto in una busta arancione. Contrariamente al solito, si presentò come una fotocopia dell'atteso comunicato numero 7:

\section{IL PROCESSO AD ALDO MORO}

Oggi 18 Aprile 1978, si conclude il periodo «dittatoriale» della D.C. che per ben trent'anni ha tristemente dominato con la logica del soppruso [sic]. In concomitanza con questa data Comunichiamo l'avvenuta ESECUZIONE del presidente della D.C. Aldo MORO; mediante "SUICIDIO". Consentiamo il recupero della salma, fornendo l'esatto luogo ove egli giace. La salma di Aldo Moro è immersa nei fondali limacciosi (ecco perché si dichiarava inpantanato [sic]) del lago Duchessa, alt. mt. 1800 circa località CARTORE (RI) zona confinante trà [sic] Abruzzo e Lazio. E' soltanto l'inizio di una lunga serie di "SUICIDI":

Il "SUICIDIO" non deve essere soltanto una "Prerogativa" del gruppo Baader Meinhof.

Inizino a tremare per le loro malefatte i vari Cossiga, Andreotti, Taviani e tutti coloro i quali sostengono il REGIME.

\footnotetext{
${ }^{30}$ Appunto per la segreteria del PCI da parte dell'avvocato Giuseppe Zupo. Cit. in DE LUTIIS, Giuseppe, Il golpe di via Fani. Protezioni occulte e connivenze internazionali dietro il delitto Moro, Milano, Sperling\&Kupfer, 2007, p. 115.

${ }^{31}$ CAP, Sentenza di primo grado, cit., pp. 257-258; X LEGISLATURA, Commissione parlamentare d'inchiesta, cit., p. 28. Pecorelli (grazie a sue personali fonti informative all'interno degli ambienti malavitosi) era certo che fosse stata proprio quella testina IBM ad aver scritto il falso comunicato n. 7. Cfr. SATTA, vladimiro, «Pecorelli, Dalla Chiesa e Moro: un intreccio da rivedere», in Nuova Storia Contemporanea, 7, 6/2003, pp. 123-154, pp. 150-152.
} 
P.S.

Rammentiamo ai vari Sossi, Barbaro, Corsi, ecc. che sono sempre sottoposti a libertà “VIGILATA".

Comunicato n. 7 18/4/1978

Per il Comunismo

BRIGATE ROSSE32

Inizialmente il nuovo documento sembrò essere identificato da Andreotti, Cossiga e dagli esperti antiterrorismo come del tutto analogo ai precedenti comunicati brigatisti ${ }^{33}$. Sebbene il comunicato fosse una fotocopia, venne quindi giudicato dagli specialisti come del tutto autentico. Tuttavia, sin dal suo ritrovamento tale documento venne accolto con scetticismo da un folto schieramento di professionisti. La prima persona che si accorse dell'evidente falsità del settimo comunicato fu il giornalista Carmine Pecorelli. Così, infatti, scrisse con sottile ironia in un suo articolo del 25 aprile: «Ricevuta la fotocopia del volantino delle "Brigate rosse" con il quale "i terroristi" comunicavano la località dove sarebbe stato abbandonato il corpo di Moro ${ }^{34}$. Sembra chiaro che le virgolette poste su «Brigate rosse» e «i terroristi» lascino intendere il fatto che per il giornalista la persona che stilò il documento non fu certamente un brigatista ${ }^{35}$. La tesi di Pecorelli è stata sostenuta anche dal capo della Divisione Investigazioni Generali e Operazioni Speciali (DIGOS) Domenico Spinella. L'ufficiale di polizia ritenne il comunicato un evidente falso a causa della fraseologia estranea a quella normale delle BR, oltre che per gli errori di ortografia e di grammatica totalmente inconsueti negli altri scritti brigatisti ${ }^{36}$ e, infine, per essere stato diffuso in una sola città e in unico esemplare piuttosto che simultaneamente in più luoghi ${ }^{37}$. Della medesima opinione fu il professor Franco Piperno ${ }^{38}$, secondo cui fu «facile riconoscere il comunicato del lago della Duchessa come redatto dai fascisti o dai servizi segreti, ma certamente non proveniente dalle BR» ${ }^{39}$. Anche il giornalista di «L'Espresso» Mario Scialoja lo ritenne sicuramente un falso, perché venne scritto «in un linguaggio che nessun

\footnotetext{
${ }^{32}$ Documento contenuto in FLAMIGNI, Gianni, La tela del ragno, cit., p. 297.

${ }^{33}$ "Continua l'angoscia: il messaggio è forse autentico ma nel lago non si riesce a trovare il corpo di Moro», in La Stampa, 19 aprile 1978.

${ }^{34}$ PECORELLI, Carmine, Le allucinanti avventure degli investigatori, «Osservatore Politico», 25 aprile 1978.

${ }^{35}$ FLAMIGNI, Sergio, Convergenze parallele. Le Brigate rosse, i servizi segreti e il delitto Moro, Milano, Kaos Edizioni, 1998, pp. 210-211.

${ }^{36}$ I cronisti sottolinearono anche gli errori di ortografia: «soppruso» è scritto con due p, «trà» è accentato, «inpantanato» con la $\mathrm{n}$ anziché con la $\mathrm{m}$. Prima d'allora, le BR non avevano mai commesso errori ortografici così grossolani. Cfr. FLAMIGNI, Gianni, La tela del ragno, cit., p. 190.

${ }^{37}$ VIII LEGISLATURA, Commissione parlamentare d'inchiesta, cit., p. 42.

${ }^{38}$ Insieme a Toni Negri aveva fondato Potere operaio, gruppo della sinistra extraparlamentare italiana attivo fra il 1969 e il 1973.

${ }^{39}$ XIII LEGISLATURA, Commissione parlamentare d'inchiesta sul terrorismo in Italia e sulle cause della mancata individuazione dei responsabili delle stragi, seduta del 18 maggio 2000, p. 13.
} 
brigatista rosso avrebbe mai usato» ${ }^{40}$. Ecco, peraltro, le valutazioni dell'agenzia «Adn Kronos» sul comunicato del lago della Duchessa:

L'autenticità del volantino lascia molti dubbi: si tratta, innanzitutto, di una fotocopia, e non di un foglio originale dattiloscritto; l'intestazione Brigate rosse è leggermente diversa nei caratteri e non risulta stampata; alcune lettere della macchina da scrivere presentano qualche diversità dai soliti caratteri: non compare il numero 1 , ed al suo posto viene utilizzata la lettera "i" maiuscola; il foglio del comunicato appare nella fotocopia visibilmente più corto di quelli solitamente adoperati; nel volantino non ci sono slogans ${ }^{41}$.

Lo stesso ex capo brigatista Mario Moretti, durante un'intervista concessa alle giornaliste Chiara Mosca e Rossana Rossanda, manifestò senza alcun dubbio la propria convinzione riguardo all'evidente falsità del documento: «Tutto di quel comunicato è falso. [...] c’è da ragionare sul perché, con quella marea di esperti, nessuno sembri accorgersi che nel comunicato che sarebbe più drammatico, quello decisivo, non una virgola somigli ai comunicati $\mathrm{Br}$, nulla nella diffusione ricordi i comunicati precedenti $»^{42}$. Infine, ad aprile il Lago della Duchessa era ricoperto da uno spesso strato di ghiaccio che avrebbe impedito a chiunque di seppellire al suo interno il cadavere del politico ${ }^{43}$.

Veniamo ora all'analisi del contenuto. Vi è un iniziale riferimento alla storia della Repubblica italiana; viene citato il trentesimo anniversario della vittoria della Democrazia cristiana alle elezioni politiche del 18 aprile 1948. Una parentesi della storia d'Italia definita dai brigatisti come «il periodo "dittatoriale"». Poi si riscontra il riferimento alla lettera che Moro inviò al ministro dell'Interno Francesco Cossiga il 29 marzo: «(ecco perché si dichiarava inpantanato) $»^{44}$. Alla fine di quella lettera

\footnotetext{
${ }^{40}$ XIII LEGISLATURA, Commissione parlamentare d'inchiesta sul terrorismo e sulle cause della mancata individuazione dei responsabili delle stragi, seduta del 14 marzo 2000, p. 3020.

${ }^{41}$ FLAMIGNI, Sergio, La tela del ragno, cit., p. 190.

${ }^{42}$ MORETTI, Mario, Brigate Rosse. Una storia italiana, Milano, Mondadori, 2012, pp. 139-140.

${ }^{43}$ Lo spesso strato di ghiaccio è ben visibile nella foto del lago nella prima pagina de «La Stampa» del 21 aprile 1978.

${ }^{44}$ Ecco il testo integrale della lettera: «A Francesco Cossiga ministro dell'Interno Caro Francesco, mentre t'indirizzo un caro saluto, sono indotto dalle difficili circostanze a svolgere dinanzi a te, avendo presenti le tue responsabilità (che io ovviamente rispetto) alcune lucide e realistiche considerazioni. Prescindo volutamente da ogni aspetto emotivo e mi attengo ai fatti. Benché non sappia nulla né del modo né di quanto accaduto dopo il mio prelevamento, è fuori discussione - mi è stato detto con tutta chiarezza - che sono considerato un prigioniero politico, sottoposto, come Presidente della D.C., ad un processo diretto ad accertare le mie trentennali responsabilità (processo contenuto in termini politici, ma che diventa sempre più stringente). In tali circostanze ti scrivo in modo molto riservato, perché tu e gli amici con alla testa il Presidente del Consiglio (informato ovviamente il Presidente della Repubblica) possiate riflettere opportunamente sul da farsi, per evitare guai peggiori. Pensare quindi fino in fondo, prima che si crei una situazione emotiva e irrazionale. Devo pensare che il grave addebito che mi viene fatto, si rivolge a me in quanto esponente qualificato della DC nel suo insieme nella gestione della sua linea politica. In verità siamo tutti noi del gruppo dirigente che siamo chiamati in causa ed è il nostro operato collettivo che è sotto accusa e di cui devo rispondere. Nelle circostanze sopra descritte entra in gioco, al di là di ogni considerazione umanitaria che pure non si può ignorare, la ragione di Stato. Soprattutto questa ragione di Stato nel caso mio significa, riprendendo lo spunto accennato innanzi sulla mia attuale condizione, che io mi trovo sotto un dominio pieno ed incontrollato, sottoposto ad
} 
Moro espresse in maniera chiarissima la sua posizione in proposito alla cosiddetta "strategia della fermezza" adottata dal Comitato di crisi: «Il sacrificio degli innocenti in nome di un astratto principio di legalità [...] è inammissibile. [...] Un atteggiamento di ostilità sarebbe un astrattezza ed un errore. Che Dio vi illumini per il meglio, evitando che siate impantanati in un doloroso episodio, dal quale potrebbero dipendere molte cose» ${ }^{45}$. Secondo il politico, il rischio di essere impantanati avrebbe riguardato i dirigenti della DC nel momento in cui non si fossero impegnati a liberarlo, appellandosi, al contrario, a un'ottusa e ingiustificata "Ragion di Stato". In questa lettera fu Moro stesso a indicare al suo partito la strada di una possibile soluzione al rapimento, invocando anche un possibile coinvolgimento del Vaticano nella trattativa: «Penso che un preventivo passo della S. Sede (o anche di altri? di chi?) potrebbe essere utile» ${ }^{46}$.

Quasi alla fine del settimo comunicato emerge una frase che sembra fuori contesto: «È soltanto una lunga serie di "suicidi": il "suicidio" non deve essere soltanto una prerogativa del gruppo Baader-Meinhof». A cosa alludono i brigatisti quando parlano di suicidio? A chi si stanno riferendo? La notte tra il 17 e il 18 ottobre del 1977, nel carcere di Stammhein (Stoccarda) si verificò la misteriosa morte dei tre capi (Andreas Baader, Gudrun Ensslin e Jan-Carl Raspe) dell'organizzazione terroristica tedesca Baader-Meinhof, denominata anche Rote Armee Fraktion. Al fine di liberarli, il 5 settembre altri terroristi appartenenti allo stesso gruppo decisero di sequestrare l'autorevole dirigente dell'Unione Cristiano-Democratica Hanns-Martin Schleyer, che fu giustiziato il giorno successivo al suicidio di Baader, Ensslin e Raspe, il 19 ottobre 1977 (esattamente sei mesi prima del 18 aprile $)^{47}$. Sembra piuttosto evidente il parallelismo tra il rapimento di Schleyer e quello di Moro.

un processo popolare che può essere opportunamente graduato, che sono in questo stato avendo tutte le conoscenze e sensibilità che derivano dalla lunga esperienza, con il rischio di essere chiamato o indotto a parlare in maniera che potrebbe essere sgradevole e pericolosa in determinate situazioni. Inoltre la dottrina per la quale il rapimento non deve recare vantaggi, discutibile già nei casi comuni, dove il danno del rapito è estremamente probabile, non regge in circostanze politiche, dove si provocano danni sicuri e incalcolabili non solo alla persona, ma allo Stato. Il sacrificio degli innocenti in nome di un astratto principio di legalità, mentre un indiscutibile stato di necessità dovrebbe indurre a salvarli, è inammissibile. Tutti gli Stati del mondo si sono regolati in modo positivo, salvo Israele e la Germania, ma non per il caso Lorenz. E non si dica che lo Stato perde la faccia, perché non ha saputo o potuto impedire il rapimento di un'alta personalità che significa qualcosa nella vita dello Stato. Ritornando un momento indietro sul comportamento degli Stati, ricorderò gli scambi tra Breznev e Pinochet, i molteplici scambi di spie, l'espulsione dei dissidenti dal territorio sovietico. Capisco che un fatto di questo genere, quando si delinea, pesi, ma si deve anche guardare lucidamente al peggio che può venire. Queste sono le alterne vicende di una guerriglia, che bisogna valutare con freddezza, bloccando l'emotività e riflettendo sui fatti politici. Penso che un preventivo passo della S. Sede (o anche di altri? di chi?) potrebbe essere utile. Converrà che tenga d'intesa con il Presidente del Consiglio riservatissimi contatti con pochi qualificati capi politici, convincendo gli eventuali riluttanti. Un atteggiamento di ostilità sarebbe una astrattezza ed un errore. Che Iddio vi illumini per il meglio, evitando che siate impantanati in un doloroso episodio, dal quale potrebbero dipendere molte cose. I più affettuosi saluti. Aldo Moro». Aldo Moro, Lettera a Francesco Cossiga, 29 marzo 1978, cit. in FLAMIGNI, Sergio, «Il mio sangue ricadrà su di loro». Gli scritti di Aldo Moro prigioniero delle Br, Milano, Kaos, 1997, pp. 57-60.

${ }^{45}$ Ibidem, p. 59-60.

${ }^{46}$ Ibidem, p. 59.

${ }^{47}$ GRIECO, Agnese, Anatomia di una rivolta. Andreas Baader, Ulrike Meinhof, Gudrun Ensslin. Un racconto a più voci, Milano, Il Saggiatore, 2010, pp. 314-335. 
Negli ambienti dell'estrema sinistra europea serpeggiò sempre l'idea che quella misteriosa e inspiegabile serie di suicidi in realtà nascondesse un omicidio di massa compiuto dalla polizia penitenziaria. In sostanza, il falso comunicato contenne una velata minaccia ai capi brigatisti in carcere che in quel momento, durante il sequestro Moro, si trovarono a Torino sotto processo ${ }^{48}$. Chi scrisse il falso comunicato volle quindi mandare un duplice messaggio al presidente della DC e ai sequestratori. Sarebbe stato Moro a ritrovarsi impantanato, e non i dirigenti del suo partito. Una risposta chiara e definitiva.

\section{Quattro piste da seguire}

Dalle indagini giudiziarie e dalle testimonianze sono emerse quattro ipotesi sul significato del falso comunicato $n .7$.

Primo: fu una prova generale da parte dello Stato per testare la reazione dell'opinione pubblica alla notizia della morte dell'onorevole Moro. Così, infatti, Eleonora Moro, moglie dello statista, ha interpretato tale documento ${ }^{49}$. Della medesima idea fu Scialoja: «Questo comunicato era stato fatto fare per vedere come avrebbe reagito l'opinione pubblica e il mondo politico di fronte alla notizia della morte di Moro: era una prova generale $»^{50}$. Supposizione ribadita anche dalla brigatista Adriana Faranda: «Quel falso comunicato ci preoccupò molto e rese inquieto anche Moro, che si chiedeva che cosa stesse succedendo. Abbiamo interpretato il falso annuncio della sua esecuzione come una prova generale di quello che sarebbe potuto accadere nel paese se Moro fosse stato effettivamente ucciso» ${ }^{51}$. Questa ipotesi venne confermata anche dall'ex brigatista Valerio Morucci all'interno del suo Memoriale: «La nostra convinzione, ed il clima non poteva permettere altra valutazione, fu che il volantino fu diffuso per anticipare e quindi far assuefare all'idea della morte di Moro». ${ }^{52}$

È certo che comunque sia Andreotti sia Cossiga non mostrarono mai una grande partecipazione per la liberazione di Moro. Anzi, la maggior parte delle volte entrambi rimasero protagonisti freddi e

\footnotetext{
${ }^{48}$ Il primo processo alle Brigate rosse si aprì il 27 maggio 1976. A causa delle reiterate minacce di morte da parte dei brigatisti imputati nei confronti di giudici, magistrati, avvocati e soprattutto giudici popolari, il processo venne posticipato fino al 10 marzo 1978 e si concluse il 23 giugno dello stesso anno. Il riferimento al processo di Torino verrebbe confermato dall'ultima riga del comunicato: «P.S. Rammentiamo ai vari Sossi, Barbaro, Corsi, ecc. che sono sempre posti a libertà vigilata». I tre personaggi citati erano il giudice Mario Sossi, rapito dalle BR il 18 aprile del 1974, e i giudici Corsi e Guido Barbaro, presidenti della Corte d'Assise del tribunale di Torino. Il messaggio suggeriva così alle BR che l'epilogo del sequestro sarebbe dovuto corrispondere a quello compiuto dalla Raf, ovvero con l'uccisione dell'ostaggio.

${ }^{49}$ VIII LEGISLATURA, Commissione parlamentare d'inchiesta, cit., p. 50.

${ }^{50}$ XIII LEGISLATURA, Commissione parlamentare d'inchiesta, 14 marzo 2000, cit., p. 3020.

${ }^{51}$ AMARA, Emmanuel, Abbiamo ucciso Aldo Moro. Dopo 30 anni un protagonista esce dall'ombra, Roma, Cooper, 2008, p. 163.

${ }^{52}$ CAVEDON, Remigio, MORUCCI, Valerio, «Memoriale Morucci-Cavedon», cit. in FLAMIGNI, Sergio, Patto di omertà. Il sequestro e l'uccisione di Aldo Moro, Milano, Kaos, 2015, p. 161.
} 
impassibili, trincerati dietro la linea della fermezza. Tutto ciò allo scopo di salvaguardare l'incolumità del popolo italiano dalla volontà eversiva dei terroristi ${ }^{53}$.

Secondo: fu una personale idea delle Brigate rosse servita a distogliere temporaneamente l'attenzione delle forze dell'ordine da Roma, al fine di permettere così ai terroristi di traslare Moro da una prigione ad un'altra più sicura. Questa opinione venne sostenuta dal brigatista Patrizio Peci, il quale dichiarò alla Commissione Moro che un esponente di Azione rivoluzionaria, Enrico Paghera, si assunse la responsabilità di redigere il falso comunicato ${ }^{54}$. Il documento sarebbe stato uno stratagemma richiesto dalle Brigate rosse ad Azione rivoluzionaria, progettato per confondere l'attenzione degli inquirenti e per consentire così ai brigatisti di uscire da Roma ${ }^{55}$. Tale ipotesi è stata avvalorata anche dal terrorista neofascista Massimo Sparti, il quale però affidò la paternità del messaggio all'amico Chicchiarelli: «Toni mi raccontò di aver preparato per scherzo e divertimento il comunicato n. 7 delle Brigate rosse per far correre le forze dell'ordine al lago della Duchessa» ${ }^{56}$. Un'ulteriore testimonianza che ha confermato tale supposizione è stata quella della moglie di Chichiarelli, Chiara Zossolo, in una sua dichiarazione resa nel 1994 al pubblico ministero di Perugia Fausto Cardella durante il processo per l'omicidio di Pecorelli:

Zossolo: «Una sera mentre eravamo a cena, no un pomeriggio vedemmo nel telegiornale tutto un servizio sulla faccenda del Lago della Duchessa e allora mio marito mi disse: "vedi tutto questo macello, tutta questa cosa, questa confusione, l'ho creata io".»

Io restai un po' meravigliata di questa cosa, dovetti credere perché lui conosceva bene il Lago della Duchessa, aveva vissuto la sua infanzia lì vicino e quindi c'erano dei riferimenti abbastanza credibili [...].

Cardella: «Le spiegò che cosa significava che lo aveva fatto lui, lo aveva provocato lui questo?» Zossolo: «Sì, questo sì, mi disse che bastava che si dicesse qualcosa alla Polizia, o alle cose, o mandasse non so un biglietto perché loro ci credessero e andassero a vedere quindi disse: basta un poco per farli muovere e fare tutto un macello.»57

In quel frangente, secondo la testimonianza di un ex agente del SISMI, Pierluigi Ravasio, le $\mathrm{Br}$ furono aiutate dalla banda della Magliana, che si occupò dell'organizzazione logistica del rapimento, riuscendo a procurare un covo sicuro per i terroristi e per Moro inizialmente presso una località compresa tra Roma e Fiumicino (Vescovio) ${ }^{58}$ e in seguito al 18 aprile in via Montalcini 8, proprio all'interno del quartiere Magliana (dove, tra l'altro, abitavano a pochi metri importanti esponenti

\footnotetext{
${ }^{53}$ SCIRÈ, Giambattista, Il caso Moro, cit., pp. 279-281.

${ }^{54}$ FLAMIGNI, Gianni, La tela del ragno, cit., p. 193.

${ }^{55}$ VIII LEGISLATURA, Commissione parlamentare d'inchiesta, cit., p. 50.

${ }^{56}$ WILLAN, Philip, I burattinai. Stragi e complotti in Italia, Napoli, Tullio Pironti, 1993, pp. 277, 279.

${ }^{57}$ Dichiarazione di Chiara Zossolo al pubblico ministero di Perugia Fausto Cardella, 11 novembre 1994, cit. in PACE, Mary, Mino Pecorelli. Il delitto irrisolto, Roma, Armando Curcio Editore, 2009, p. 221.

${ }^{58}$ BISCIONE, Francesco M., Il delitto Moro. Strategie di un assassinio politico, Roma, Editori Riuniti, 1998, p. 152.
} 
dell'organizzazione criminale $)^{59}$. Una zona molto più sicura per i sequestratori e allo stesso tempo meno controllata dalle forze dell'ordine perché territorio della banda ${ }^{60}$. Il coinvolgimento della malavita romana nell'Affaire Moro è stato ulteriormente confermato da Raffaele Cutolo in una sua dichiarazione, resa nel 1993 al giudice istruttore di Roma Otello Lupacchini: «A dire di Nicolino Selis, la prigione del parlamentare democristiano si trovava nei pressi di un appartamento che egli teneva come nascondiglio per eventuali latitanze». ${ }^{61}$

Terzo: fu il compimento di un'operazione depistatoria (progettata dai servizi segreti con o senza l'autorizzazione delle istituzioni) finalizzata a mettere sotto pressione le Brigate rosse durante le trattative per il rilascio dell'onorevole. Secondo il magistrato Ferdinando Imposimato e l'avvocato della DC Giuseppe De Gori, si sarebbe dovuto escludere il fatto che le Brigate rosse avessero potuto decidere autonomamente fin dall'inizio la sorte di $\mathrm{Moro}^{62}$, perché il comunicato del lago della Duchessa fu la firma consapevole dello Stato sulla sua sentenza di condanna a morte ${ }^{63}$. Se Moro non fosse morto allora lo Stato avrebbe eliminato non soltanto i brigatisti detenuti a Torino, ma anche gli stessi sequestratori ${ }^{64}$. Un'altra conferma sull'idea che il comunicato del Lago della Duchessa fosse stato progettato dalle istituzioni è stata data dall'ex brigatista Enrico Fenzi in un'intervista rilasciata al giornalista Sergio Zavoli: «Secondo le Brigate rosse, il comunicato del Lago della Duchessa era un falso del governo, della polizia, insomma del potere [...] ed era il segnale chiaro e inequivocabile che nessuna trattativa era possibile [...] che lo Stato non avrebbe mai trattato per Moro» ${ }^{65}$. L'opinione di Fenzi venne condivisa anche dall'ex brigatista Anna Laura Braghetti:

\footnotetext{
${ }^{59}$ Ad esempio, uno dei capi della banda, Nicolino Selis, abitava proprio in via Montalcini. Pochi giorni prima dell'esecuzione di Moro, il parlamentare democristiano Flaminio Piccoli si era anche con Selis nell'estremo tentativo di salvare la vita del suo collega e amico. Cfr. XIV LEGISLATURA, Commissione parlamentare d'inchiesta sul fenomeno della criminalità organizzata mafiosa o similare, Relazione conclusiva, Doc. XXIII, No. 16, Tomo II, 20 gennaio 2006, p. 881. Un altro boss della banda, Abbruciati, abitava in via Fuggetta 59, a cento passi da via Montalcini. Cfr. DE LUTIIS, Giuseppe, Il golpe di via Fani, cit., p. 104.

${ }^{60}$ XI LEGISLATURA, Commissione parlamentare d'inchiesta sul fenomeno della mafia e sulle altre associazioni criminali similari, Relazione conclusiva, Doc. XXIII, No. 14, 12 aprile 1994, pp. 216-217.

${ }^{61}$ Dichiarazione di Raffaele Cutolo al giudice istruttore di Roma Otello Lupacchini, 12 novembre 1993. Cit. in TRIBUNALE PENALE DI ROMA, Sentenza-ordinanza del giudice istruttore di Roma Otello Lupacchini, 13 agosto 1994, p. 79.

${ }^{62}$ Imposimato: «Non è vero affatto [...] che le Brigate rosse avevano deciso di eliminare Moro fin dal suo sequestro. Il 6 maggio le Brigate rosse avevano rinviato di 3 giorni l'esecuzione di Moro per poter trattare con lo Stato la sua liberazione». Del resto, quale tipo di vantaggi avrebbero potuto avere le BR uccidendolo? Nessuno. Tutto ciò avrebbe causato soltanto conseguenze deleterie per la propria propaganda. Cfr. XIII LEGISLATURA, Commissione parlamentare d'inchiesta sul terrorismo in Italia e sulle cause della mancata individuazione dei responsabili delle stragi, seduta del 24 novembre 1999, p. 2595.

${ }^{63}$ XIII LEGISLATURA, Commissione parlamentare d'inchiesta sul terrorismo in Italia e sulle cause della mancata individuazione dei responsabili delle stragi, seduta dell'8 luglio 1998, p. 1591.

${ }^{64} \mathrm{Col}$ passare dei giorni brigatisti abbassarono le pretese durante le trattative per la liberazione dell'ostaggio: inizialmente con lo scambio di tredici prigionieri contro uno, poi uno contro uno e infine solo la richiesta d'attenuazione dei rigori del regime carcerario. Cfr. XIII LEGISLATURA, Commissione parlamentare d'inchiesta sul terrorismo in Italia e sulle cause della mancata individuazione dei responsabili delle stragi, Decisioni adottate dalla Commissione nella seduta del 22 marzo 2001 in merito alla pubblicazione degli atti e dei documenti prodotti e acquisiti, Doc. XXIII, No. 64, Vol. I, Tomo VI, 22 marzo 2001, p. 148.

${ }^{65}$ ZAVOLI, Sergio, La notte della Repubblica, Milano, Mondadori, 2015, p. 303.
} 
Pensava[m]o che il falso comunicato fosse una manovra dei servizi per farci capire che lo Stato non avrebbe trattato, e che Moro era perduto. Non ci sfuggì il tono del messaggio apocrifo: era cinico e beffardo, non il nostro stile. Anche un bambino avrebbe potuto accorgersene, ma nessuno in quelle ore convulse si era impegnato più di tanto per vagliarne l'autenticità 66 .

Sempre secondo Imposimato il falso comunicato non fu altro che «una creazione della banda della Magliana per indurre le Brigate rosse ad uccidere l'ostaggio» ${ }^{67}$. La malavita romana avrebbe quindi agito «per conto dei servizi segreti, che a loro volta si mossero per ordine di qualche politico» ${ }^{68}$. Servizi segreti che, tuttavia, secondo quanto affermato da Richard Gardner, ambasciatore statunitense in Italia tra il 1977 e il 1981, brancolarono nel buio per tutta la durata della trattativa: «non ci si può immaginare in quale stato fossero i servizi segreti in Italia a quel tempo: disorganizzazione totale. Non avevano nemmeno un fascicolo sui capi delle Brigate Rosse» ${ }^{69}$. Una circostanza alquanto misteriosa se si ricorda il fatto che già dal 19 marzo la questura di Roma era in grado di identificare ben ventidue brigatisti, alcuni dei quali appartenenti al commando di Via $\mathrm{Fani}^{70}$. Tutto ciò può far pensare soltanto che non vi fosse stata, volutamente o no, una reciproca collaborazione tra gli organi di sicurezza nazionale. Quindi il documento potrebbe essere interpretato come un messaggio mandato alle Brigate rosse su come si sarebbe dovuto concludere il sequestro, perché «la quasi certezza che Moro stesse facendo dichiarazioni importanti è stata la ragione dell'operazione lago della Duchessa. [...] misura presa per bloccare le rivelazioni che Moro sicuramente stava facendo» ${ }^{71}$. Questa teoria venne, infine, avvalorata sia dal direttore del quotidiano «La Repubblica» Eugenio Scalfari che dalla testimonianza diretta dall'ex brigatista Alberto Franceschini durante una sua intervista rilasciata al giornalista Gianni Minoli per il programma televisivo «Mixer» nel $1987^{72}$. Molto probabilmente i servizi segreti utilizzarono il falso comunicato come un avviso (e allo stesso tempo una minaccia) nei confronti delle BR riguardo alla sorte di Moro, ma anche per ciò che concernette la rischiosa pubblicazione delle sue carte inerenti ai maggiori scandali dell'Italia repubblicana ${ }^{73}$.

\footnotetext{
${ }^{66}$ BRAGHETTI, Anna Laura, TAVELLA, Paola, Il prigioniero, Milano, Feltrinelli, 2005, p. 150.

${ }^{67}$ AMARA, Emmanuel, Abbiamo ucciso Aldo Moro, cit., p. 165.

${ }^{68}$ Ibidem.

${ }^{69}$ WEINER, Tim, CIA. Ascesa e caduta dei servizi segreti più potenti del mondo, Milano, Rizzoli, 2010, p. VII.

${ }^{70}$ LANARO, Silvio, Storia dell'Italia repubblicana. L'economia, la politica, la cultura, la società dal dopoguerra agli anni '90, Venezia, Marsilio, 2007, p. 463.

${ }^{71}$ Cfr. XIII LEGISLATURA, Commissione parlamentare d'inchiesta, 1999, cit., p. 2627.

${ }^{72}$ SCIRÈ, Giambattista, Il caso Moro, cit., p. 284.

${ }^{73}$ GALLI, Giorgio, Piombo rosso. La storia completa della lotta armata in Italia dal 1970 a oggi, Milano, Baldini Castoldi Dalai, 2007, pp. 119-121. Nel memoriale di Aldo Moro si fa riferimento a sedici temi: il Piano Solo, la strategia della tensione, la riforma dei servizi segreti (ottobre 1977), i finanziamenti della DC, il prestito del Fondo Monetario Internazionale al governo (1977), lo scandalo Lockheed, l'accordo sulla fiducia DC-PCI, gli ambasciatori statunitensi a Roma, la presidenza della Repubblica, il rinnovamento della DC, Umberto Agnelli e il Senato (1976), la nomina di Giuseppe Medici alla presidenza del gruppo industriale Montedison (1977), la DC
} 
Quarto: fu la realizzazione, da parte dello Stato, di una contromossa inaspettata e studiata, avente lo scopo di determinare confusione all'interno delle Brigate rosse, che fino a quel giorno avevano gestito completamente le trattative per la liberazione dell'ostaggio attraverso i sei comunicati precedenti. Il sostituto procuratore di Roma Luciano Infelisi dichiarò dinanzi alla Commissione Moro il 27 gennaio 1981: «Nei giorni precedenti [al 18 aprile] qualcuno aveva proposto che i servizi segreti scrivessero delle lettere, dei volantini, al fine di creare una reazione. [...] Se i nostri servizi, sollecitati, non erano all'altezza di farlo, allora mi domando chi può avere creato il volantino in quel particolare momento». ${ }^{74}$ Soltanto nel corso delle inchieste giudiziarie inerenti all'omicidio di Pecorelli, il 10 maggio 1993, il magistrato Claudio Vitalone dichiarò che il falso comunicato venne progettato "per costringere le $\mathrm{Br}$ a tenere in vita l'ostaggio», suggerendo, inoltre, l'idea che «con l'intervento degli organi di polizia giudiziaria, [...] si potesse far diramare un comunicato apocrifo per disorientare le $\mathrm{Br} »^{75}$.

Le quattro interpretazioni formulate sulla vicenda potrebbero essere definitivamente chiarificate riportando quella testimonianza che ho ritenuto come la più rilevante, perché sostenuta da uno dei principali e indiscussi protagonisti di questa vicenda: Stephen R. Pieczenik, ex capo dell'Ufficio per la gestione dei problemi del terrorismo internazionale del Dipartimento di Stato a Washington DC e consigliere speciale del ministro Cossiga all'interno del Comitato di crisi durante la parentesi del sequestro:

Bisognava preparare l'opinione pubblica italiana e quella europea ad un eventuale decesso di Moro e per questo è stata definita quella che viene chiamata una "operazione psicologica". Questa operazione consisteva nella pubblicazione di un falso comunicato nel quale era annunciata la morte di Moro ed era indicato il luogo dove il suo corpo poteva essere ritrovato. È tutto quello che so, perché non ho partecipato direttamente alla messa in atto di questa operazione, che avevamo deciso nel comitato di crisi.

Le Brigate rosse [...] non si aspettavano di trovarsi di fronte ad un altro terrorista che li utilizzava e li manipolava psicologicamente con lo scopo di prenderli in trappola. Avrebbero potuto venirne fuori facilmente, ma erano stati ingannati. Ormai non potevano fare altro che uccidere Moro. Questo è il grande dramma di questa storia ${ }^{76}$.

Giunto in Italia come esperto antiterrorista subito dopo la strage di via Fani, Pieczenik si accorse della tragica fine del sequestrato già dopo le prime lettere di Moro e i primi comunicati delle Brigate

e le banche, il ruolo antisovietico di Gladio, Cossiga e le forze dell'ordine, la DC e la stampa nazionale. Cfr. BISCIONE, Francesco M. (a cura di), Il memoriale di Aldo Moro rinvenuto in via Monte Nevoso a Milano, Roma, Coletti, 1993, pp. 45-96.

${ }^{74}$ GOTOR, Miguel, Il memoriale della repubblica. Gli scritti di Aldo Moro dalla prigionia e l'anatomia del potere italiano, Milano, Mondadori, 2011, p. 317.

${ }^{75}$ XIII LEGISLATURA, Commissione parlamentare d'inchiesta, 2001, cit., p. 190.

${ }^{76}$ AMARA Emmanuel, Abbiamo ucciso Aldo Moro, cit., p. 166. 
rosse che si susseguirono durante i cinquantacinque giorni. Il messaggio di quei documenti e la costante renitenza del Comitato di crisi a trattare con i terroristi lo resero ben consapevole del fatto che, da parte di entrambi, non vi sarebbe stata la minima intenzione di salvare la vita dell'ostaggio ${ }^{77}$. $\mathrm{Fu}$ proprio Pieczenik a confermare il suo presentimento di una congiura organizzata dai colleghi di Moro, specialmente quelli nominati da Cossiga all'interno del Comitato:

Ci fu una cosa che emerse in maniera chiarissima, e che mi sbalordì. Io non conoscevo l'uomo Aldo Moro, dunque desideravo farmi un'idea di che persona fosse e di quanta resistenza avesse. Ci ritrovammo in questa sala piena di generali e di uomini politici, tutta gente che lo conosceva bene, e [...] alla fine ebbi la netta sensazione che a nessuno di loro Moro stesse simpatico o andasse a genio come persona, Cossiga compreso. Era lampante che non stavo parlando con i suoi alleati 7 .

Profondamente vicino alle machiavelliche manovre politiche di Kissinger, Pieczenik si propugnò come il massimo esponente della "strategia dell'inattività", ovvero dell'intrattabilità con i terroristi in ogni caso. Attraverso questa modalità d'azione il Comitato di crisi avrebbe annichilito, almeno politicamente, la figura di Moro, evitando il rischio del "compromesso storico" e valorizzando il PSI di Bettino Craxi in una futura fase di transizione politica, collocata temporalmente tra gli anni della violenza terroristica e gli anni del crollo del comunismo in Unione Sovietica ${ }^{79}$. E così, in effetti, avvenne. Ecco perché, secondo il giornalista statunitense Robert Katz, venne scelto proprio Pieczenik per quel delicato ruolo di intermediario:

È particolarmente significativo che non sia stato Brian Jerkins, che pure è uno degli esperti mondiali di terrorismo più ascoltati, ad essere convocato a Roma, bensì Steve Pieczenik. A differenza della linea di Jerkins, abbastanza flessibile, la tesi avanzata da Pieczenik fu la seguente: «Bisogna dimostrare che nessuno è indispensabile per la sopravvivenza dello statonazione». [...] L’idea era quindi di dimostrare che si poteva fare a meno persino di Aldo Moro ${ }^{80}$.

Anche Pecorelli scrisse, in un suo articolo intitolato Vergogna buffoni! e pubblicato su «OP» il 16 gennaio 1979, della figura di Pieczenik e del suo ruolo di coordinatore all'interno del Comitato di crisi. Dopo essere ritornato negli Stati Uniti, Pieczenik confermò al Congresso di aver dato a Cossiga le migliori disposizioni per poter riuscire a salvare la vita di Moro, con la promessa (da parte di Pecorelli) di pubblicare ulteriori rivelazioni inerenti alla morte dello statista democristiano nei

\footnotetext{
${ }^{77}$ SCIRÈ, Giambattista, Il caso Moro, cit., p. 276.

${ }^{78}$ FLAMIGNI, Sergio, Convergenze parallele, cit., pp. 158-159.

${ }^{79}$ GALLONI, Giovanni, 30 anni con Moro, Roma, Editori Riuniti, 2008, p. 249.

${ }^{80} \mathrm{KATZ}$, Robert, I giorni dell'ira. Il caso Moro senza censure, Roma, Adnkronos, 1982, p. 260.
} 
fascicoli seguenti. La sera del 20 marzo 1979 Pecorelli venne ucciso davanti alla sede del suo periodico ${ }^{81}$.

\section{Conclusioni}

Il falsario romano Antonio Chichiarelli venne probabilmente incaricato dai servizi segreti per redigere un falso comunicato brigatista che annunciasse l'avvenuta morte di Moro e il suo seppellimento nel lago della Duchessa. Per di più, secondo quanto scritto negli elaborati presentati nel 2001 dalla Commissione parlamentare sul terrorismo in Italia, fu proprio il senatore e magistrato Vitalone che propose al sostituto procuratore generale di Roma Infelisi l'idea della produzione di un falso comunicato brigatista. Oltre a ciò Vitalone non escluse la possibilità di un coinvolgimento di alcuni organi di polizia, che avrebbero dovuto provvedere alla ricerca dell'esecutore. Un organo di polizia avrebbe potuto assicurare meglio il collegamento tra i servizi segreti (che in tal modo sarebbero stati protetti da possibili indagini) e i criminali, esecutori materiali della manovra depistatoria. Secondo quanto sostenuto da Vitalone, sarebbe stato sufficiente infiltrare tra le BR personaggi appartenenti alla criminalità e al neofascismo per destabilizzare i piani dei terroristi. $\mathrm{E}$ di soggetti criminali Vitalone ne conosceva, secondo quanto venne riferito il 7 maggio 1994 da Fabiola Moretti, compagna di Abbruciati, che in certe occasioni fece da chaffeur per Vitalone negli incontri segreti di quest'ultimo con il boss della banda della Magliana Enrico De Pedis, in quel tempo latitante $^{82}$. Una figura oscura, quella del magistrato Vitalone, pervasa, secondo la Zossolo, di un «falso perbenismo»" ${ }^{83}$ e inserita tra il mondo della politica e il mondo della criminalità. Un personaggio che secondo la Moretti risultò protagonista di uno scambio di regali con De Pedis:

L'attenzione deve soffermarsi sui regali fatti o ricevuti da Claudio Vitalone e precisamente sul regalo di un servizio per manicure fatto da Claudio Vitalone a Enrico De Pedis, sul regalo di un anello fatto da Claudio Vitalone a Fabiola Moretti e sul regalo di un orologio Rolex fatto da Enrico De Pedis a Claudio Vitalone e sugli omaggi di pesce da parte di Enrico de Pedis a Claudio Vitalone ${ }^{84}$.

Uno di questi protagonisti appartenenti alla malavita romana reclutati da Infelisi potrebbe essere stato proprio Chichiarelli, che aveva conoscenze interne ai servizi di sicurezza e alle forze

\footnotetext{
${ }^{81}$ PECORELLI, Carmine, «Vergogna buffoni!», OP-Osservatore Politico, 16 gennaio 1979, cit. in GALLONI, Giovanni, 30 anni con Moro, cit., p. 250.

${ }^{82}$ TRIBUNALE PENALE DI ROMA, Sentenza-ordinanza, cit., pp. 168-169.

${ }^{83}$ Dichiarazione di Chiara Zossolo al pubblico ministero di Perugia Fausto Cardella, 11 novembre 1994, cit. in PACE, Mary, Mino Pecorelli, cit., p. 223.

${ }^{84}$ CAP, Sentenza di primo grado, cit., pp. 427-428.
} 
dell'ordine ${ }^{85}$. Considerato il fatto che la polizia giudiziaria venne sempre reputata come il braccio operativo della magistratura inquirente, sarebbe stato quindi tale reparto quello maggiormente idoneo a rintracciare gli uomini adatti per un'operazione tanto delicata, all'interno dell'unico ambiente in cui si sarebbero potuti reperire: quello della malavita. Chichiarelli non sembrò essere mai direttamente collegato ad alcun corpo di polizia o servizio di sicurezza, ma frequentò spesso un suo amico intimo, Dal Bello, che, come è stato affermato precedentemente, era stato in quel periodo un informatore al servizio del maresciallo Antonio Solinas, personaggio interno al Nucleo di Polizia giudiziaria in Roma. In questo caso Solinas seppe dal suo confidente Dal Bello già durante il periodo del sequestro Moro del ruolo di Chichiarelli nell'operazione depistatoria del falso comunicato. Tuttavia ciò venne rivelato da Solinas agli inquirenti soltanto in seguito alla morte di Chichiarelli, avvenuta il 28 settembre 1984, quindi sei anni dopo il sequestro Moro. Solinas riferì la notizia soltanto ai due ufficiali del SISDE Massimo Erasmo e Giuseppe Scipioni, che pur sapendo preferirono tacere per nascondere la verità alla magistratura ${ }^{86}$. Il fatto che lo Stato abbia potuto accreditare come autentico un palese falso, oltre alla gravità della vicenda, è di grandissima importanza storica, oltre che giuridica, in quanto ciò potrebbe implicare che Chichiarelli stesse agendo per conto dei servizi segreti in un'operazione che, purtroppo, ebbe l'appoggio e la complicità delle forze dell'ordine $e^{87}$.

\footnotetext{
${ }^{85}$ CASTRONOVO, Manlio, Vuoto a perdere. Le Brigate Rosse, il rapimento, il processo e l'uccisione di Aldo Moro, Nardò, BESA Editrice, 2007, p. 207.

${ }^{86}$ DE LUTIIS, Giuseppe, Il golpe di via Fani, cit., pp. 115-116.

${ }^{87}$ CAP, Sentenza di primo grado, cit., p. 254; XIII LEGISLATURA, Commissione parlamentare d'inchiesta, 2001, cit., pp. 190-191.
} 


\section{L'AUTORE}

Francesco LANDOLFI Francesco Landolfi si è laureato nel 2014 in Scienze Storiche presso l'Università di Padova. Nel 2016 ha conseguito un master in Criminologia Critica e Sicurezza Sociale presso le Università di Padova e Bologna. Attualmente è dottorando di ricerca in Studi Storici presso le Università di Firenze e Siena con un progetto di ricerca sui collegamenti illeciti tra la politica locale e la mafia italo-americana a New York durante gli anni del proibizionismo (1920-1933).

URL: < http://www.studistorici.com/progett/autori/\#Ladndolfi > 